\title{
A case of Sotos syndrome presented with end-stage renal disease due to the posterior urethral valve
}

\author{
Won Im Cho', Jung Min Ko ${ }^{1,2 *}$, Hee Gyung Kang ${ }^{1,2,3}$, II-Soo Ha, and Hae II Cheong ${ }^{1,2,3}$ \\ ${ }^{1}$ Department of Pediatrics, Seoul National University Children's Hospital, Seoul, Korea \\ ${ }^{2}$ Research Coordination Center for Rare Diseases, Seoul National University Hospital, Seoul, Korea \\ ${ }^{3}$ Kidney Research Institute, Medical Research Center, Seoul National University College of Medicine, Seoul, Korea
}

Sotos syndrome (SS, OMIM 117550) is characterized by prenatal and postnatal overgrowth with multiple congenital anomalies. However, there have been few cases of growth retardation caused by renal failure from infancy. We report a case of dysplasia of the bilateral kidneys with renal failure and poor postnatal growth. A 2-month-old boy visited the emergency room owing to poor oral intake and abdominal distension. He was born at the gestational age of 38 weeks with a birth weight of 4,180 g. After birth, he had feeding difficulty and abdominal distension. Upon physical examination, his height and weight were in less than the 3rd percentile, while his head circumference was in the 50th percentile on the growth curve. He also showed a broad and protruding forehead and high hairline. Blood laboratory tests showed severe azotemia; emergent hemodialysis was needed. Abdominal ultrasonography revealed bilateral renal dysplasia with multiple cysts and diffuse bladder wall thickening. A posterior urethral valve was suggested based on vesicoureterography and abdominal magnetic resonance findings. Results of a colon study to rule out congenital megacolon did not reveal any specific findings. The conventional karyotype of the patient was $46, \mathrm{XY}$. Array comparative genomic hybridization study revealed a chromosome 5 q35 microdeletion including the NSD1 gene, based on which SS was diagnosed. We describe a case of SS presenting with end stage renal disease due to posterior urethral valve. The typical somatic overgrowth of SS in the postnatal period was not observed due to chronic renal failure that started in the neonatal period.

Key words: NSD1, Overgrowth, Sotos syndrome, Posterior urethral valve, Chronic kidney failure.

\section{Introduction}

Sotos syndrome (SS, OMIM 117550) is an autosomal dominantly inherited congenital malformation syndrome characterized by four cardinal features: pre- and postnatal overgrowth, typical facial dysmorphism, macrocephaly, and variable degrees of mental retardation [1].SS patients sometimes show additional clinical features including neonatal jaundice, congenital heart defects, brain anomalies, neonatal hypotonia, skeletal anomalies, and increased incidence of malignancy [2]. Among them, genitourinary defects such as vesicoureteral reflux, multiple renal cysts, unilateral renal aplasia, and hypoplasia have been associated with about 15\% of SS patients, with vesico-ureteral reflux being the most commonly observed [2].

Received: 12 November 2014, Revised: 28 November 2014, Accepted: 4 December 2014, Published: 31 December 2014

${ }^{*}$ Corresponding author: Jung Min Ko, M.D., Ph.D.

Department of Pediatrics, Seoul National University Children's Hospital, Seoul National University College of Medicine, 101 Daehak-ro, Jongno-gu, Seoul 110-769, Korea.

Tel: +82-2-2072-3570, Fax: +82-2-743-3455, E-mail: jmko@snu.ac.kr

Conflict of interest: We declare that we do not have any conflicts of interests.

(c) This is an open-access article distributed under the terms of the Creative Commons Attribution Non-Commercial License (http://creativecommons.org/licenses/by-nc/3.0/) which permits unrestricted non-commercial use, distribution, and reproduction in any medium, provided the original work is properly cited.

(c) Copyright 2014 by the Korean Society of Medical Genetics 
Kurotaki et al. [3] reported a de novo translocation $t(5,8)$ $(q 35 ; q 24.1)$ in SS that bisected the NSD 1 (nuclear receptorbinding SET domain containing protein) gene on chromosome 5q35.3. Several other groups have also confirmed that alterations in NSD 1 cause SS. In Japan and Korea, microdeletion of chromosome $5 q 35$ is the most common cause of SS [4]. However, $5 q 35$ microdeletions are uncommon in Caucasian populations, accounting for only 10\% of affected individuals [5]. The phenotypes of SS patients with NSD 1 intragenic mutations and of those with $5 q 35$ microdeletion differ since some of the features of SS, such as overgrowth and learning disability, are attributable to NSD1, whereas others, such as cardiac and renal anomalies, are more commonly associated with the deletion of neighboring genes in the $5 q 35$ region rather than NSD 1 itself [4].

Since the first description by Sotos et al. in 1964 (requoted from reference [1]), hundreds of cases of SS have been reported to date, with an estimated incidence of 1/15,000-1/20,000 [6]. However, the prevalence of SS in Korea has not yet been reported, and there have only been seven reports of the disease in Korea [6-12]. Previous studies have reported that only a few patients presented with mild hydronephrosis with or without vesico-ureteral reflux. There have been no reports of SS with end stage renal disease requiring hemodialysis due to posterior urethral valve in Korea.

In this report, we describe the first patient with $5 q 35$ microdeletion who required renal replacement therapy at the age of 2 months due to end stage renal disease.

\section{Case}

A 2-month-old boy visited the emergency room of our hospital due to poor oral intake and abdominal distension. The patient was born by caesarian section performed at a local hospital at 38+0 weeks of gestation with a birth weight of 4,180 $g$ (>97th percentile) and length of $52 \mathrm{~cm}$ (75th percentile). He was the parents' first child. Oligohydramnios was observed during pregnancy, and fetal ultrasonography revealed a distended fetal bladder and abdomen. Amniocentesis was performed at 18 weeks of gestation, at which time a normal male karyotype, $46, X Y$, was revealed by chromosomal analysis of the amniotic cells. At the age of 14 days, he showed poor sucking power and difficulty feeding with abdominal distension and visited another hospital where he was admitted for 25 days. Blood laboratory findings showed elevated levels of blood urea nitrogen (83.9 mg/dL), serum creatinine $(9.93 \mathrm{mg} / \mathrm{dL})$, and uric acid (12.8 mg/dL). The karyotype of peripheral blood leukocytes was 46, XY. Abdominal sonography revealed multiple cysts in both kidneys, and renal cortical uptake of both kidneys was not visualized on dimercaptosuccinic acid scan. Abdominal distension and feeding intolerance persisted. Therefore, a colon study was performed; however, there was no evidence of Hirschsprung's disease.

Upon the first visit to our hospital, the patient's body length was $52.8 \mathrm{~cm}(<3 \mathrm{rd}$ percentile), with a weight of $3.95 \mathrm{~kg}(<3 \mathrm{rd}$ percentile) and head circumference of $37 \mathrm{~cm}$ (50th percentile). He had a blood pressure of $69 / 41 \mathrm{mmHg}$, pulse rate of 122 beats/ $\mathrm{min}$, respiratory rate of 48 breaths/min, and body temperature of $37^{\circ} \mathrm{C}$. The patient had a long face with a broad and prominent forehead, pointed chin, and down-slanted eyes. He also showed a high arched palate and pectus excavatum deformity of the thorax. The left testis was undescended, and generalized hypotonia was found upon neurologic examination. Although a cardiac murmur was not audible, a small muscular ventricular septal defect (2.5 mm in size) was detected by echocardiography. In the laboratory findings, elevated levels of blood urea nitrogen of $63 \mathrm{mg} / \mathrm{dL}$ and serum creatinine of $6.20 \mathrm{mg} / \mathrm{dL}$ were noted. Therefore, emergent hemodialysis was performed. Urine analysis performed to evaluate tubular function showed $\mathrm{N}$-acetyl- $\beta$ glucosaminidase (NAG) levels of $1.6 \mathrm{mg} / \mathrm{dL}(0.3-11.5 \mathrm{IU} / \mathrm{L})$, $\mathrm{NAG} /$ creatinine of $37.1 \mathrm{IU} / \mathrm{g} \cdot \mathrm{Cr}(0-5.6 \mathrm{IU} / \mathrm{g} \cdot \mathrm{Cr})$, and tubular reabsorption of phosphate of $85 \%$ (>85\%), based on which combined renal tubulopathy was suspected.

Vesicoureterography was performed to identify the cause of neonatal onset renal failure, and dilatation of the bladder neck and posterior urethra were observed, causing urethral obstruction to be suspected (Fig. 1A). Abdominal magnetic resonance (MR) images also revealed bilateral tortuously dilated ureters and multiple small cysts in both kidneys (Fig. 1B). Brain MR images showed only mild ventriculomegaly.

Based on the facial dysmorphism and congenital anomalies of multiple organs, we decided to perform an additional array comparative genomic hybridization analysis. The results showed a $1.85 \mathrm{Mb}$ deletion on chromosome $5 q 35.2 q 35.3$, harboring NSD 1 (Fig. 2A). Fluorescence in situ hybridization analysis was performed for confirmation of the deletion (Fig. 2B), and the diagnosis of SS caused by 5 q35 microdeletion was made.

Renal replacement therapy using regular hemodialysis (3 times a week) was performed for end stage renal failure, and the blood urea nitrogen was decreased. The patient is now 24 months old, and catch-up of growth in both height $(82.5 \mathrm{~cm}$, 
(A)

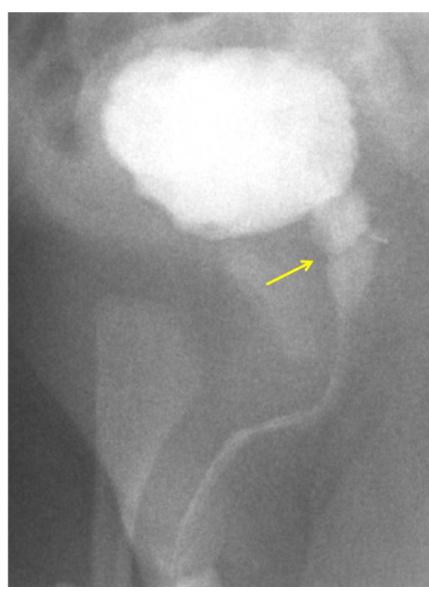

(B)

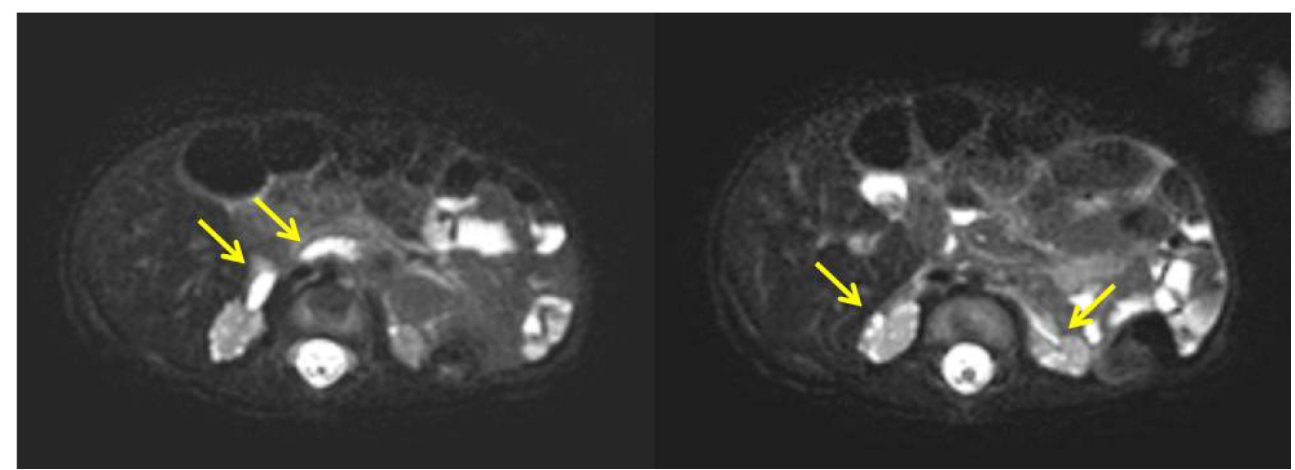

Fig. 1. (A) Vesicoureterography showed linear filling defects (arrow) between the dilated bladder neck and posterior urethra. The dilated posterior urethra was funnel-shaped, and the bladder wall was thickened. (B) Tortuous and dilated ureters (arrows) in both sides and a thickened bladder wall were observed in the abdominal magnetic resonance images of the patient. Bilateral small-sized kidneys with multiple small cysts can also be seen.

(A)

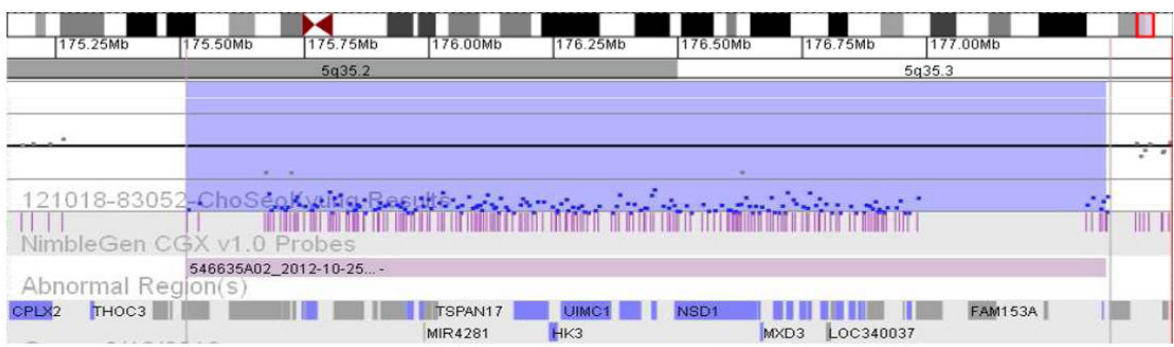

(B)

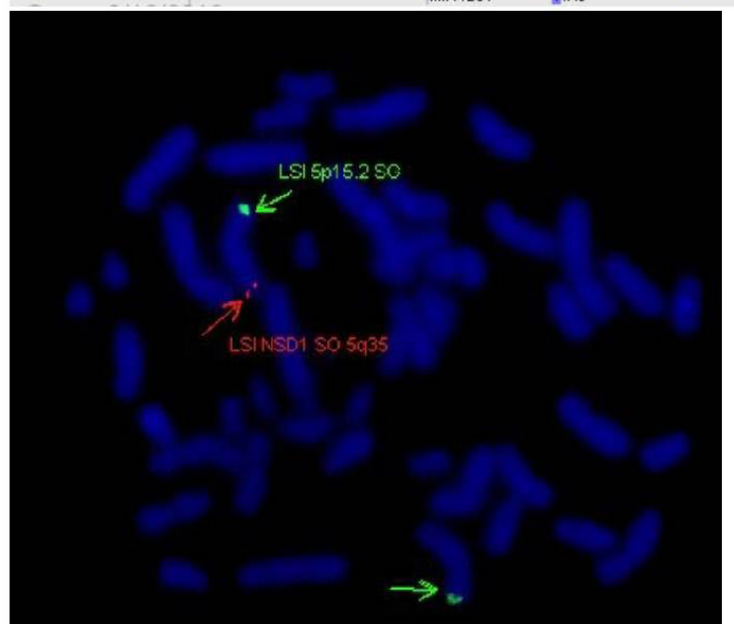

Fig. 2. Results of aCGH (A) and FISH (B) analyses revealed a $1.85 \mathrm{Mb}$ deletion in 5q35.2q35.3. aCGH, array comparative genomic hybridization; FISH, fluorescence in situ hybridization.

5th-10th percentile) and weight (10.6 kg, 5th-10th percentile) was observed with renal replacement therapy. Macrocephaly with a head circumference of $48 \mathrm{~cm}$ (50th-75th percentile) was evident. In addition, his developmental milestones are generally delayed. He can control his head perfectly and roll over to both sides, but he cannot sit alone at the age of 24 months. Regular hemodialysis was changed to peritoneal dialysis at the age of 17 months, and rehabilitation therapy has been continued to date.

\section{Discussion}

Our patient showed the characteristic facial features of SS including frontal bossing, antimongoloid slant of the palpebral fissures, sparseness of hair in the frontoparietal region, high anterior hairline, and prominent and pointed jaw, as previously described [1]. In particular, macrosomia and macrocephaly 
are considered cardinal manifestations that can be important clues for the clinical diagnosis of SS [1]. The patient herein was born large for his gestational age with a large head, and this finding had persisted since the initial antenatal sonographic examination. Additionally, the patient's birth weight was over the 97th percentile for his age and gender. However, upon admission to our hospital at the age of 2 months, his length was only $52.8 \mathrm{~cm}$ ( $<3 \mathrm{rd}$ percentile), weight was only $3.95 \mathrm{~kg}(<3 \mathrm{rd}$ percentile), and head circumference was $37 \mathrm{~cm}$ (50th percentile). These observations might be associated with rapid progression of chronic renal failure after birth. SS patients also usually have abnormal neurologic manifestations such as developmental delays, hypotonia, myoclonus, and seizures [2]. The majority of SS patients have various degrees of developmental delay. Walking and speech development are generally delayed, and most patients have various degrees of intellectual disabilities with intelligence quotients ranging from below 30 to above 100 [5]. In addition, seizures have been reported in up to $50 \%$ of SS patients [1]. Consistent with previous studies, hypotonia and seizure-like movements were observed in the patient, accompanied by developmental delays in motor and neuropsychological function.

The pectus excavatum presented in the patient is also a common feature of SS, though the thoracic wall anomalies observed are not a cardinal feature. Thoracic wall anomalies such as pectus excavatum are seen in 2-5\% of individuals with SS [13]. About 20\% of SS patients have cardiac anomalies, of which atrial septal defects and patent ductus arteriosus are most common [13]. The patient examined herein also had a ventricular septal defect. In addition, the patient had difficulty feeding, with abdominal distension and defecation difficulty that mimicked Hirschsprung's disease in the neonatal period. Early feeding difficulty is a common gastrointestinal symptom, along with neonatal jaundice. The frequency of gastrointestinal symptoms observed in SS patients is unclear. However, a previous study reported that about $40 \%$ of SS patients required tube feeding during infancy due to feeding difficulty [1].

Genitourinary anomalies associated with SS were identified in 19\% of patients and can be accompanied by duplex or vesico-ureteric reflux, pelvo-ureteric junction obstruction, and cystic kidneys [14]. However, vesico-ureteric reflux is the most common urogenital problem in SS [4], and unilateral renal agenesis is often observed $[5,15]$. There were no abnormal kidney findings detected by antenatal sonography in the patient. However, renal sonography performed due to the finding of azotemia in laboratory tests showed bilateral renal dysplasia and multiple cortical cysts, suggesting a posterior urethral valve at the age of 2 months. Although there was one report of a Japanese patient who had multiple dysplastic renal cysts in the unilateral kidney, the other kidney was normal, making renal replacement therapy unnecessary [4]. In the patient herein, bilateral renal dysplasia due to posterior urethral valve was observed, which may have caused chronic renal failure to start during the fetal period. During pregnancy, oligohydramnios had been noted. This finding also suggested that bilateral renal dysplasia and renal function deterioration had started at that time [16]. Therefore, renal replacement therapy was required at the age of 2 months. Although SS is commonly accompanied by urogenital anomalies, severe bilateral renal dysplasia and rapid progression to end-stage renal disease caused by posterior urethral valve is quite rare.

NSD1 on chromosome $5 q 35$ is the main causative gene of SS [5] and is expressed in several tissues including the brain, kidney, skeletal muscle, spleen, lung, and thymus [17]. The haploinsufficiency of NSD 1 occurs in 60-90\% of clinically diagnosed SS patients and can be transmitted in an autosomal dominant fashion, but over 95\% of patients gain SS from de novo mutation without any family history [6]. NSD 1 abnormalities include microdeletion of $5 q 35$ and intragenic mutations within the NSD 1 gene [6]. 5q35 microdeletion encompassing NSD 1 is more common (approximately 50\%) than NSD 1 intragenic mutation in Japanese patients with SS; however, less than 10\% of non-Japanese patients show $5 q 35$ microdeletion [18]. A previous report documenting Korean patients with SS indicated that 53\% of patients had a $5 q 35$ microdeletion, which was consistent with the findings of Japanese studies [12]. The patient herein was also diagnosed as having SS caused by $5 q 35$ microdeletion. In previous studies, some genotype-phenotype correlations have been suggested. The frequency of cardiac and urogenital malformations in the $5 q 35$ deletion group was reported to be more common than in the NSD1 intragenic mutation group $[4,5]$. Saugier-Veber et al. [19] described 116 patients with NSD 1 alterations, in which a significantly higher frequency of cardiac (37.5\% vs. 8\%) and renal ( $50 \%$ vs. $12 \%$ ) malformations were observed in the $5 q 35$ deletion group than in the group with the NSD 1 intragenic mutation. The patient herein also had a $5 q 35$ microdeletion with both cardiac and urologic anomalies.

Here, we reported the first case of an infant with SS presenting with severe uremia associated with chronic renal failure. At diagnosis, the patient showed lower body weight and short stature due to chronic renal failure, even though he had SS. 
Therefore, if patients have a history of relative macrocephaly, high birth weight, and long birth length, SS should not be excluded for differential diagnosis of multiple congenital malformation syndrome.

\section{Acknowledgements}

This study was supported by grant no. NRF-2012R1A1A3001588 from the Korean Ministry of Science, ICT, and Future Planning.

\section{References}

1. Cole TR, Hughes HE. Sotos syndrome: a study of the diagnostic criteria and natural history. J Med Genet 1994;31:20-32.

2. Vilchis Z, Najera N, Pérez-Duran J, Najera Z, Gonzalez L, del Refugio Rivera $M$, et al. The high frequency of genetic diseases in hypotonic infants referred by neuropediatrics. Am J Med Genet A 2014;164:1702-5.

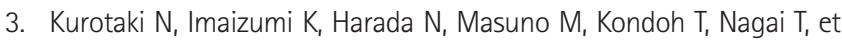
al. Haploinsufficiency of NSD1 causes Sotos syndrome. Nat Genet 2002;30:365-6.

4. Nagai T, Matsumoto N, Kurotaki N, Harada N, Niikawa N, Ogata T, et al. Sotos syndrome and haploinsufficiency of NSD1: clinical features of intragenic mutations and submicroscopic deletions. J Med Genet 2003;40:285-9.

5. Tatton-Brown K, Douglas J, Coleman K, Baujat G, Cole TR, Das S, et al; Childhood Overgrowth Collaboration. Genotype-phenotype associations in Sotos syndrome: an analysis of 266 individuals with NSD1 aberrations. Am J Hum Genet 2005;77:193-204.

6. Ko JM. Genetic syndromes associated with overgrowth in childhood. Ann Pediatr Endocrinol Metab 2013;18:101-5.

7. Cho EH, Park BY, Cho JH, Kang YS. Comparing two diagnostic laboratory tests for several microdeletions causing mental retardation syndromes: multiplex ligation-dependent amplification vs fluorescent in situ hybridization. Korean J Lab Med 2009;29:71-6.

8. Lim JJ, Yoon SH. The first neurosurgical analysis of 8 Korean children with Sotos syndrome. J Korean Neurosurg Soc 2008:44:240-4.

9. Kim SB, Yang S, Kim HD, Oh PS, Cha JK, Shin JH. A case of cerebral gigantism (Sotos syndrome). J Korean Soc Pediatr Endocrinol 2002;7:122-7.

10. Kim IS, Kim JH, Choi YY, Ma JS, Hwang TJ. A case of Sotos syndrome. J Korean Pediatr Soc 1995;38:725-9.

11. Ahn HJ, Kim YT, Seol IH, Shin JH. Two cases of cerebral gigantism (Sotos syndrome). J Korean Pediatr Soc 1990;33:1153-6.

12. Sohn YB, Lee CG, Ko JM, Yang JA, Yun JN, Jung EJ, et al. Clinical and genetic spectrum of 18 unrelated Korean patients with Sotos syndrome: frequent $5 q 35$ microdeletion and identification of four novel NSD1 mutations. J Hum Genet 2013;58:73-7.

13. Tatton-Brown K, Rahman N. Clinical features of NSD1-positive Sotos syndrome. Clin Dysmorphol 2004;13:199-204.

14. Thomas A, Lemire EG. Sotos syndrome: antenatal presentation. Am J Med Genet A 2008;146A:1312-3.

15. Su PH, Yu JS, Chen SJ, Chen JY, Tsao TF. Persistent falcine sinus and unilateral renal agenesis in a girl with Sotos syndrome. Clin Dysmorphol 2011;20:42-6.

16. Chen CP. Prenatal findings and the genetic diagnosis of fetal overgrowth disorders: Simpson-Golabi-Behmel syndrome, Sotos syndrome, and Beckwith-Wiedemann syndrome. Taiwan J Obstet Gynecol 2012;51:186-91.

17. Kurotaki N, Harada N, Yoshiura K, Sugano S, Niikawa N, Matsumoto $\mathrm{N}$. Molecular characterization of NSD1, a human homologue of the mouse Nsd1 gene. Gene 2001;279:197-204.

18. Faravelli F. NSD1 mutations in Sotos syndrome. Am J Med Genet C Semin Med Genet 2005;137C:24-31.

19. Saugier-Veber $P$, Bonnet $C$, Afenjar A, Drouin-Garraud V, Coubes $C_{\text {, }}$ Fehrenbach $S$, et al. Heterogeneity of NSD1 alterations in 116 patients with Sotos syndrome. Hum Mutat 2007;28:1098-107. 